\title{
Métricas da Informação sobre Gestão Ambiental na Pecuária
}

\author{
Metrics of Information on Environmental Management in Livestock
}

\section{Métricas de Información Sobre Gestión Ambiental en Ganadería}

\author{
Alice Munz Fernandes ${ }^{1}$ \\ Guilherme Cunha Malafaia ${ }^{2}$ \\ Odilene de Souza Teixeira ${ }^{3}$ \\ Francisca Viviane dos Santos ${ }^{4}$
}

\begin{abstract}
Resumo: O objetivo desta pesquisa foi caracterizar as publicações científicas sobre gestão ambiental na atividade pecuária a partir de métricas da informação. Para isso, empregou-se uma análise cientométrica pautada em técnicas de bibliometria e sociometria. A partir de uma busca na base de dados Web of Science, considerando como período de publicações todos os anos limitado até agosto de 2019, obteve-se um portfólio composto por 105 artigos científicos. Para operacionalizar a análise dos dados e possibilitar a organização e formações de redes utilizaram-se os softwares VOSviewer e UCINET. Os resultados obtidos demonstraram que o portfólio de estudos está distribuído entre 82 journals e revistas científicas distintas. Também se constatou que nos últimos cinco anos o interesse dos pesquisadores sobre a temática também se intensificou, respondendo por aproximadamente $42 \%$ do total das publicações. Para a análise das cocitações considerou-se como unidade analítica os autores citados que possuíam ao menos seis vínculos de cocitação, resultando em 34 autores dentre os 3.951 identificados. A vista disso formaram-se três clusters e 59 ligações, distribuídos da seguinte forma: (i) formado por nove autores; (ii) formado por três autores, e; (iii) formado por dois autores. Portanto, verifica-se a maximização do interesse dos pesquisadores sobre o assunto ao longo dos últimos anos, bem como as extensas redes de colaboração, inclusive interinstitucional e internacional, a partir das quais os estudos são desenvolvidos.
\end{abstract}

Palavras-chave: Análise Cientométrica. Impacto Científico. Redes de Colaboração.

\begin{abstract}
The objective of this research was to characterize the scientific publications on environmental management in livestock activity based on metrics of information. For this, a scientometric analysis based on bibliometric and sociometric techniques was used. From a search of the Web of Science database, considering the publication period all limited years until August 2019, a portfolio of 105 scientific articles was obtained. The software VOSviewer and UCINET were used to operationalize the data analysis and enable the organization and network formation. The results showed that the study portfolio is distributed among 82 distinct scientific journals. It was also found that in the last five years, the interest of researchers on the subject has also intensified, accounting for approximately $42 \%$ of the total of publications. For the analysis of cocitations, the authors mentioned who had at least six links of cocitation were considered as the analytical unit, which resulted in 34

Universidade Federal do Rio Grande do Sul (UFRGS), Porto Alegre, RS, Brasil, alicemunz@gmail.com

Empresa Brasileira de Pesquisa Agropecuária (EMBRAPA), Campo Grande, Brasil, gcmalafaia@gmail.com

Universidade Federal do Rio Grande do Sul (UFRGS), Porto Alegre, RS, Brasil, odilene rs@ @hotmail.com

Universidade Federal do Rio Grande do Sul (UFRGS), Porto Alegre, RS, Brasil,

francisca_viviane@yahoo.com.br
\end{abstract}


authors out of the 3,951 identified. Given this, three clusters and 59 links were formed, distributed as follows: (i) formed by nine authors; (ii) formed by three authors; and (iii) formed by two authors. Therefore, the interest of researchers on the subject has been maximized over the last years, as well as the extensive networks of collaboration, including inter-institutional and international, from which the studies are developed.

Keywords: Scientific Analysis. Scientific Impact. Collaboration Networks.

Resumen: El objetivo de esta investigación fue caracterizar las publicaciones científicas sobre gestión ambiental en la actividad ganadera con base en métricas de información. Para esto, se utilizó un análisis cienciométrico basado en técnicas bibliométricas y sociométricas. A partir de una búsqueda en la base de datos de Web of Science, considerando un período limitado de publicación de cada año hasta agosto de 2019, se obtuvieron un total de 105 artículos científicos. Para operacionalizar el análisis de los datos y permitir la organización y formación de redes se utilizó el software VOSviewer y UCINET. Los resultados mostraron que los artículos estudiados se distribuyeron entre 82 periódicos y revistas científicas diferentes. También se descubrió que en los últimos cinco años el interés de los investigadores en el tema también se ha intensificado, representando aproximadamente el $42 \%$ del total de publicaciones. Para el análisis de las cocitaciones se consideró como unidad analítica que los autores mencionaron que tenían al menos seis enlaces de cocitación, resultando en 34 autores de los 3.951 identificados. En vista de esto, se formaron tres grupos y 59 enlaces, distribuidos de la siguiente manera: (i) formado por nueve autores; (ii) formado por tres autores, y; (iii) formado por dos autores. Por lo tanto, se logra validar el creciente interés de los investigadores sobre el tema en los últimos años, como también las amplias redes de colaboración, incluidas las interinstitucionales e internacionales, a partir de las cuales se desarrollan los estudios.

Palabras clave: Análisis científico. Impacto científico Redes de colaboración.

\section{INTRODUÇÃO}

A atividade pecuária está passando por mudanças globais, principalmente no que concerne ao modelo de produção dos animais, pois a pecuária extrativista foi gradativamente substituída por estabelecimentos de grande porte que exploram produtos cárneos e lácteos, principalmente. Dessa forma, o modelo de negócio é direcionado para a produção em escala, com maior número de animais por área, o que consequentemente trouxe consigo as preocupações referentes aos impactos ambientais, essencialmente no que se refere ao uso dos recursos naturais, solo e água (ZANONI, 2004).

Em termos de sociedade, essas inquietações convertem-se em exigências de uma produção mais limpa e sustentável (RAMANKUTTY et al., 2018). Desse modo, os gestores rurais e os stakeholders das cadeias produtivas agroalimentares estão buscando atender as exigências legais por meio da gestão dos recursos naturais sem comprometer seus aspectos econômicos e financeiros (PALHARES, 2007).

No entanto, independente do modelo de produção, a gestão ambiental deve ser incorporada para que as organizações atinjam seu potencial tanto social e econômico quanto 
e-ISSN: 2317-675X | Comun. \& Inf., Goiânia, GO, v. 24, p. 1-16, 2021.

ambiental. Ou seja, considerando as preocupações emergentes da sociedade contemporânea, sem a devida adequação ambiental, as empresas não conseguem permanecer ativas. Essa interpretação também condiz com os sistemas de produção pecuários, principalmente após a segunda guerra mundial, quando estes sistemas passaram por mudanças expressivas (GIANNI; GOTZAMANI; TSIOTRAS, 2017).

Não obstante, no âmbito científico a proposição conjunta de tais temáticas - gestão ambiental e pecuária - a tem permeado distintas áreas do conhecimento, sobretudo devido aos crescentes desafios concernentes a segurança alimentar, crescimento populacional e aquecimento global, por exemplo (FAO, 2017). Logo, apesar das origens de tal linha de investigação ser reportada às ciências agrárias, animais e sociais tem-se mecanismos provenientes da ciência da informação que contribuem para a identificação do comportamento científico desta temática (HUAI; CHAI, 2016).

Ante ao exposto, a pesquisa realizada teve como objetivo caracterizar as publicações científicas sobre gestão ambiental na atividade pecuária a partir de métricas da informação. Assim, a cientometria, enquanto área do conhecimento, possibilita identificar certos padrões da literatura publicada, bem como analisar o comportamento de um conjunto de conhecimentos (SENGUPTA, 1992). Logo, demostra-se pertinente uma análise sobre tal perspectiva, haja vista a multidisciplinariedade da temática investigada, bem como suas distintas interfaces e circunscrições.

\section{GESTÃO AMBIENTAL NA ATIVIDADE PECUÁRIA}

O cenário de intensificação da produção pecuária, com maior concentração de animais por área ocorreu de forma exponencial e global nos últimos anos. Esse incremento na escala de produção é mediado pelo crescimento da população humana, o que reflete na maximização da demanda por alimentos. No entanto, esse contexto trouxe preocupações inerentes a degradação ambiental e consequentemente ameaças à produtividade pecuária a longo prazo (RAMANKUTTY et al., 2018). O uso demasiado dos recursos naturais, principalmente a exploração da terra e da água, bem como o manejo inadequado de resíduos na produção animal e as emissões de gases poluentes, os ditos gases de efeito estufa, como o metano, são recorrentes e polêmicos quanto a produção animal e a conformidade ambiental (MOURI; AISAKI, 2015; LIJÓ et al., 2018; ALEKSANDR et al., 2019; SOUZA FILHO et al., 2019).

Com o objetivo de mitigar os riscos dos impactos ambientais na pecuária surgem alternativas tecnológicas baseadas na pecuária de precisão, a qual visa monitorar e gerenciar a 
e-ISSN: 2317-675X | Comun. \& Inf., Goiânia, GO, v. 24, p. 1-16, 2021.

produção automaticamente. Assim, a pecuária torna-se mais econômica, social e ambientalmente sustentável a partir da observação individual dos animais (TULLO; FINZI; GUARINO, 2019).

Além disso, métodos agroecológicos para a produção animal (RAMANKUTTY et al., 2018), sistemas integrados de produção lavoura-pecuária (SOUZA FILHO et al., 2019), rotações de culturas (WANG et al., 2019) e aplicação de estudos de modelagem e simulação para identificar os possíveis impactos ambientais dos sistemas de produção configuram-se como mecanismos inovadores para a produção de alimentos com reduzidos impactos ambientais (MOURI; AISAKI, 2015).

Destarte, com vistas a práticas pecuárias sustentáveis, ou seja, por meio da intensificação sustentável, tem-se aspectos relacionados a implementação de sistemas dotados de maior rigidez na avaliação e licenciamento ambiental para as produções bovinas no Brasil - o que já existe para a indústria de aves e de suínos.

Em âmbito mundial, a política ambiental da Rússia e da Europa, por exemplo, está passando por um período de transição para a inserção dos princípios de Melhores Técnicas Disponíveis (MTD), que preveem que empresas com alto impacto, como aquelas que exploram a atividade pecuária de forma intensiva, sejam obrigadas a adquirir a licença ambiental integrada até 2025 (ALEKSANDR et al., 2019). Conquanto, a China também está passando por modificações, a partir das quais os pesquisadores apontam que regulamentos rigorosos seriam úteis para controlar a descarga de poluentes, bem como o fornecimento de subsídios para o tratamento de estrume (QIAN et al., 2018).

A gestão ambiental e as práticas de manejo nas indústrias são tópicos debatidos cientificamente no Brasil. No entanto, o país apresenta um baixo índice de desenvolvimento sustentável (PINTO et al., 2018). Dessa forma, identifica-se que propostas de melhorias do uso dos recursos naturais, bem como o conhecimento sobre a gestão ambiental existem, mas essas questões ainda são aparentemente apresentadas de forma teórica e com reduzida aplicabilidade prática.

\section{METODOLOGIA}

A pesquisa realizada configura-se como quantitativa no que concerne à sua abordagem (BRYMAN, 1988) e descritiva em relação a sua finalidade (LAKATOS; MARCONI, 2011). Como procedimento técnico empregou-se a cientometria que corresponde ao "estudo da mensuração e quantificação do progresso científico" (SILVA; BIANCHI, 2001, p. 05). 
e-ISSN: 2317-675X | Comun. \& Inf., Goiânia, GO, v. 24, p. 1-16, 2021.

Para Milojevic e Leydesdorff (2013), tanto a cientometria, quanto a bibliometria, infometria e webometria são consideradas pertencentes a uma mesma área de investigação que apresenta métodos e técnicas similares, cujo objeto corresponde a informações já publicadas. Assim, os autores enfatizam que esse conjunto de procedimentos metodológicos se referem a métricas da informação, também chamadas de iMetrics.

Assim, a análise realizada pauta-se em aspectos concernentes à bibliometria, que corresponde a "uma área de estudo quantitativo da comunicação escrita, que aplica um conjunto de métodos e técnicas modernas de formalização matemática e estatística" (PEHAR, 2010, p. 1) e a própria sociometria que aborda aspectos de interação social (BORDONS et al., 2015).

Este tipo de investigação busca averiguar a existência de padrões em determinado conjunto de literatura (GENG et al., 2017), configurando-se como uma ramificação da ciência da informação relacionado à estatística (HUAI; CHAI, 2016). Estes modelos ou padrões de comportamento da comunicação escrita observados ao longo dos anos são expressos por meio das basilares leis da bibliometria quais sejam: Lei de Lotka, Lei de Bradford e Lei de Zipf (HOOD; WILSON, 2001).

A Lei de Lotka é também conhecida como Lei do Quadrado Inverso e determina que um reduzido número de autores é responsável por uma elevada quantidade de publicações científicas acerca de determinado assunto, ao passo que um grande número de autores apresenta pouca contribuição ou representatividade sobre esta mesma temática (CHUNG; COX, 1990).

Conquanto, a Lei de Bradford ou Lei da Dispersão pressupõem que os periódicos que possuem o maior número de publicações sobre determinado assunto correspondem aqueles que teoricamente apresentam um núcleo de relevância e qualidade superior nesta mesma área do conhecimento científico (BRADFORD, 1934; ROUSSEAU; ROUSSEAU, 2000). Por sua vez, a Lei de Zipf ou Lei do Mínimo Esforço parte da frequência de ocorrência de palavras no texto, promovendo a identificação dos termos dotados de maior relevância sobre determinada temática (ADAMIC; HUBERMAN, 2002).

Conquanto, a fim de verificar a colaboração entre os autores empregou-se também elementos pertinentes à sociometria, que se fundamenta na relevância das redes e interações de pesquisa na produção do conhecimento científico (BORDONS et al., 2015). Assim, temse o agrupamento de indivíduos com o intuito de colaboração múltipla, o que, em essência, não se configura como um fenômeno recente (GUTIÉRREZ et al., 2016). 
De acordo com Blumberg e Hare (1999), a sociometria enquanto procedimento de investigação tem sido utilizada de distintas formas e em diferentes áreas, o que inclui sua pertinência para a seleção de pessoal dotado de maior qualificação, processamento de informações, avaliação de desempenho, contribuição ao processo decisório, e complementação de conhecimentos.

A partir de tais delineamentos, definiu-se como orientação de busca a existência dos termos "environmental management" e "livestock", mediados pelo operador booleano "and", no título, resumo e/ou palavras-chaves. A base de dados escolhida para a busca consistiu na Web of Science, cujo período de publicações correspondeu a todos os anos limitados até a data de 05 de agosto de 2019.

Definiu-se também como critério de inclusão/exclusão a tipologia de documento correspondente a artigo. Sendo assim, o portfólio de estudos analisados foi composto por 105 documentos. Para análise dos dados, empregou-se um conjunto de técnicas considerando a diversidade de variáveis que caracterizam o portfólio de estudos. Assim, aspectos como ano de publicação, periódico e autores foram analisados por meio de frequência relativa e absoluta e comparados com os postulados pelas leis da bibliometria.

Posteriormente, elaboraram-se redes de cocitação e de colaboração entre os autores, expressando a estrutura intelectual da referida temática (KHASSEH; SOHEILI; CHELK, 2018). Para tanto, empregaram-se técnicas inerentes a bibliometria moderna (THOMPSON; WALKER, 2015), viabilizadas por meio dos softwares VOSviewer e UCINET, respectivamente.

\section{ANÁLISE E DISCUSSÃO DOS RESULTADOS}

A partir do portfólio obtido, inicialmente verificou-se a distribuição temporal das publicações científicas sobre gestão ambiental e pecuária, o que demonstrou que apesar de não haver uniformidade no que se refere ao número de publicações em função do tempo, é visível a maximização do interesse dos pesquisadores sobre a temática nos últimos anos. A Figura 1 ilustra tal distribuição e apresenta uma linha de tendência crescente.

Figura 1 - Distribuição temporal das publicações

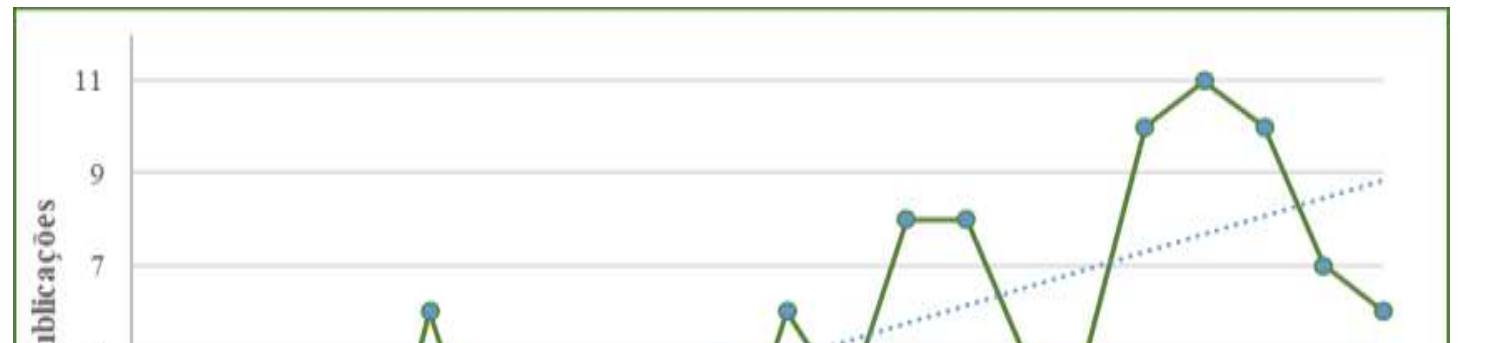


Fonte: Resultados da pesquisa (2019).

Observa-se que os últimos cinco anos respondem por aproximadamente $42 \%$ do total de publicações sobre gestão ambiental e pecuária, o que enfatiza a relevância de tal temática no meio científico. Para Silva (2012) este fenômeno deve-se a intensificação das preocupações globais acerca dos impactos ambientais provenientes das atividades agrícolas, o que vai ao encontro dos pilares da sustentabilidade (ELKINGTON, 2012).

No tocante aos periódicos, evidencia-se que o portfólio de publicações analisadas está distribuído entre 82 journals e revistas científicas distintas. Estes, em geral, são considerados de alto impacto, haja vista que $77 \%$ dos artigos estão contidos em periódicos com Journal Citation Report (JCR) superior a 1,00. Tal métrica expressa a relevância relativa das revistas científicas onde a temática investigada é publicada (GARFIELD, 2006), ou seja, está disponível em periódicos com representativos índices de citação (PODSAKOFF et al., 2005).

Conquanto, tem-se também que $6 \%$ dos periódicos respondem por cerca de $20 \%$ dos artigos sobre gestão ambiental e pecuária, o que reflete o postulado pela Lei de Bradford e, portanto, podem ser considerados como aqueles que, teoricamente, possuem maior relevância para esta área do conhecimento (BRADFORD, 1934; ROUSSEAU; ROUSSEAU, 2000). As características principais deste conjunto de periódicos estão descritas no Quadro 1.

Quadro 1 - Principais periódicos

\begin{tabular}{|l|l|l|l|}
\hline Periódico & Publicações & JCR & Editoração \\
\hline
\end{tabular}


e-ISSN: 2317-675X | Comun. \& Inf., Goiânia, GO, v. 24, p. 1-16, 2021.

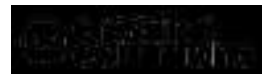

\begin{tabular}{|c|c|c|c|}
\hline $\begin{array}{c}\text { Journal of Environmental } \\
\text { Management }\end{array}$ & 7 & 4,865 & $\begin{array}{c}\text { R. Dewil (KU Leuven), } \\
\text { J. M. Evans (Stetson University), B. } \\
\text { Tansel (Florida International } \\
\text { University) }\end{array}$ \\
\hline $\begin{array}{c}\text { Agricultural Systems } \\
\text { Australian Journal of } \\
\text { Experimental Agriculture }\end{array}$ & 3 & 4,131 & $\begin{array}{c}\text { G. Martin (French National Institute } \\
\text { for Agricultural Research) }\end{array}$ \\
\hline $\begin{array}{c}\text { Environmental Management } \\
\text { Environmental Modelling \& } \\
\text { Software }\end{array}$ & 3 & 2,376 & $\begin{array}{c}\text { W. Bryden (University of } \\
\text { Queensland) }\end{array}$ \\
\hline \begin{tabular}{c} 
B. W. Brooks (Baylor University) \\
\hline
\end{tabular} & 3 & 4,552 & $\begin{array}{c}\text { D. P. Ames (Brigham Young } \\
\text { University) }\end{array}$ \\
\hline
\end{tabular}

Fonte: Resultados da pesquisa (2019).

Não obstante, sob o enfoque científico, a literatura tende a ser organizada conforme suas especialidades, o que permite inferir que os documentos científicos apresentam certa similaridade quando são citados pelos mesmos artigos (KOROM, 2019). Assim, para a análise das cocitações, considerou-se como unidade analítica os autores citados que possuem ao menos seis vínculos de cocitação.

Deste modo, tem-se 34 autores dentre os 3.951 identificados pelo software VOSviewer que atendem a tal critério. Contudo, deste montante, o maior conjunto de itens que apresentam alguma conexão é formado por 14 itens. Logo, estes representam os 14 vértices da estrutura de rede apresentada na Figura 2.

Figura 2 - Rede de cocitações

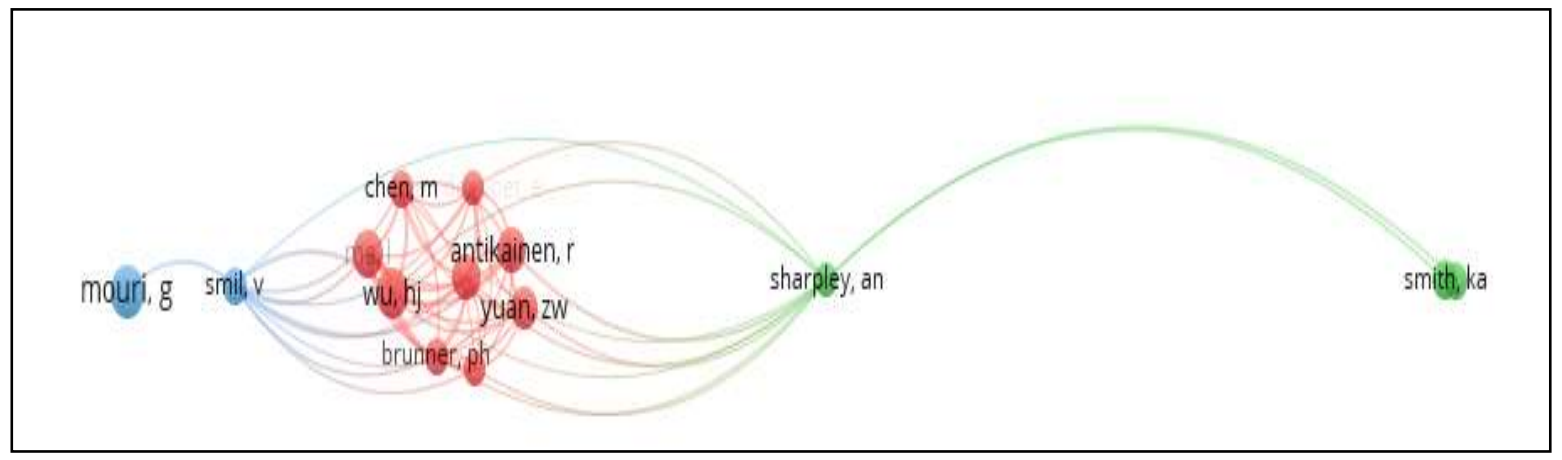

Fonte: Elaborado pelos Autores, com o auxílio do software VOSviewer (2019).

Destaca-se que o tamanho dos rótulos e seus respectivos círculos possui uma relação diretamente proporcional à força total das ligações, o que torna alguns marcadores invisíveis para evitar sobreposição (VAN ECK; WALTMAN, 2019). A coloração das ligações é definida pelos clusters ou grupos aos quais os autores pertencem e proximidade entre cada 
e-ISSN: 2317-675X | Comun. \& Inf., Goiânia, GO, v. 24, p. 1-16, 2021.

vértice (autor) demonstra frequência com a qual tais referências tendem a ser citadas conjuntamente nos estudos analisados (KOROM, 2019).

Assim, tem-se um total de três clusters e 59 ligações, distribuídos da seguinte forma: (i) cluster vermelho: formado por nove autores; (ii) cluster verde: formado por três autores, e; (iii) cluster azul: formado por dois autores. Destaca-se que o autor $\mathrm{H}$. J. Wu - afiliado a School of Earth and Environment of Anhui University os Science and Technology - integrante do cluster vermelho caracteriza-se como aquele que apresenta a maior força entre as ligações (184), sendo responsável por 13 citações. Isto é, trata-se do autor que é citado de forma associada à uma gama de outros autores.

Em contrapartida, G. Mouri - Earth Observation Data Integration \& Fusion Research Initiative, The University of Tokyo - pertencendo ao cluster azul configura-se como o autor com o maior número de citações (15), mas com baixo nível de força entre as ligações (10). Ou seja, este configura-se como um autor que, apesar de ser frequentemente citado, não apresenta um padrão de associação com outros pesquisadores quanto a composição de referências.

No tocante aos termos predominantes nos artigos analisados, tem-se que mesmo que exista uma heterogeneidade de sinônimos e variações para o mesmo termo, identificar aqueles dotados de maior recorrência nas investigações científicas configura-se como uma maneira de identificar o domínio de diferentes áreas do conhecimento (GRIFFITHS; STEYVERS, 2004).

Assim, para operacionalizar tal identificação e posterior análise, elaborou-se uma rede de coocorrência de termos baseada em dados textuais, empregando o VOSviewer. Para isso, utilizaram-se os dados contidos nas palavras-chaves, haja vista sua relevância para a indexação dos estudos e sua utilização na busca de informações e delimitação de campo científico (BRANDAU; MONTEIRO; BRAILE, 2005).

Logo, definiu-se como número mínimo de quatro coocorrência, sendo que dos 894 termos, somente 24 atenderam à este critério. Assim, estes vértices estão distribuídos em quatro clusters e apresentam 104 ligações, com força total de associação correspondente a 154. A Figura 3 ilustra esta rede de coocorrência das palavras-chaves predominantes. 
Figura 3 - Rede de coocorrência de palavras-chaves

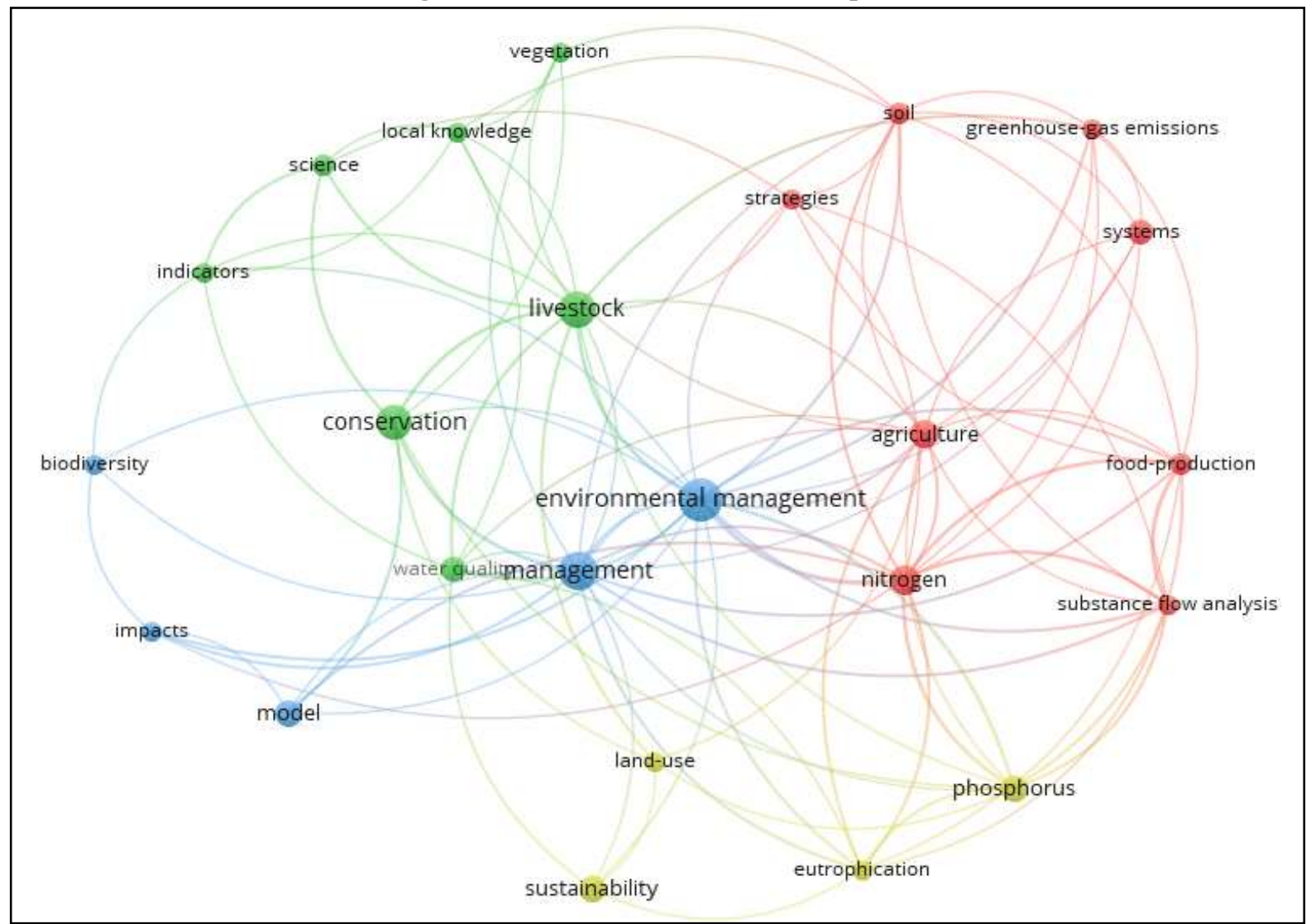

Fonte: Elaborado pelos Autores, com o auxílio do software VOSviewer (2019).

Observa-se que o cluster vermelho é o maior de todos, sendo composto por oito vértices. Além disso, tendo em vista as palavras-chaves que o integram, pode-se inferir que a este pertencem os artigos com ênfase em estratégias e sistemas de produção de alimentos orientados pelas preocupações ambientais.

Conquanto, o cluster verde, formado por sete vértices, ilustra aspectos concernentes a conservação dos recursos naturais, envolvendo qualidade da água e conhecimento local, por exemplo. Por sua vez, o cluster azul, composto por cinco vértices, caracteriza-se por compilar estudos com enfoque gerencial, ou seja, que abordam a construção de modelos e elementos voltados à biodiversidade e aos impactos, bem como a própria gestão ambiental propriamente dita.

Por fim, o cluster amarelo possui quatro vértices e apresenta um apelo mais técnico, isto é, envolve questões relacionadas a mudança de uso da terra, elementos químicos presentes no solo, fenômenos naturais e seu reflexo na sustentabilidade dos modos de produção agrícola. Destarte, a partir do portfólio analisado, constata-se a existência de certos padrões quantificáveis no que tange a determinação de termos em estudos científicos, indo ao 
e-ISSN: 2317-675X | Comun. \& Inf., Goiânia, GO, v. 24, p. 1-16, 2021.

encontro do postulado pela Lei de Zipf (ADAMIC; HUBERMAN, 2002). Deste modo, identificar os termos descritores dotados de maior recorrência, bem como a similaridade e associação entre eles demonstra-se pertinente para o entendimento da temática gestão ambiental e pecuária enquanto campo científico que permeia distintas áreas do conhecimento.

Não obstante, quanto aos indivíduos que respondem pela autoria dos artigos científicos analisados, verifica-se que as 105 investigações foram elaboradas ou desenvolvidas por 401 pesquisadores com distintas afiliações. Todavia, cerca de 97\% dos autores contribuem com somente uma publicação, ao passo que $2 \%$ dos pesquisadores respondem por $23 \%$ do total de investigações. Este quadrado inverso demonstra que um número restrito de indivíduos se configura como os principais autores ou especialistas em determinada temática, corroborando com o pressuposto pela Lei de Lotka (CHUNG; COX, 1990).

$\mathrm{O}$ pesquisador que apresenta o maior número de estudos sobre gestão ambiental e pecuária é o chinês Z. W. Yuan afiliado ao State Key Laboratory of Pollution Control and Resource Reuse, School of the Environment, Nanjing University. Seus estudos envolvem a Avaliação do Ciclo de Vida de diferentes sistemas de produção animal, bem como o impacto da dieta dos ruminantes na emissão de gases de efeito estufa ou quantidades de fósforo no solo, por exemplo.

Contudo, a colaboração entre os autores configura-se como um eficaz meio de maximizar os atributos positivos dos pesquisadores e aumentar a contribuição da investigação para a ciência, sobretudo quando se trata de uma temática que permeia distintas áreas do conhecimento (GUTIÉRREZ et al., 2016). Assim, para ilustrar as diferentes interações quantificáveis entre os autores (nós) (BARABASI et al., 2002), apresenta-se na Figura 4 a rede de colaboração entre os pesquisadores que apresentavam no mínimo cinco associações. 
Figura 4 - Rede de colaboração entre os autores

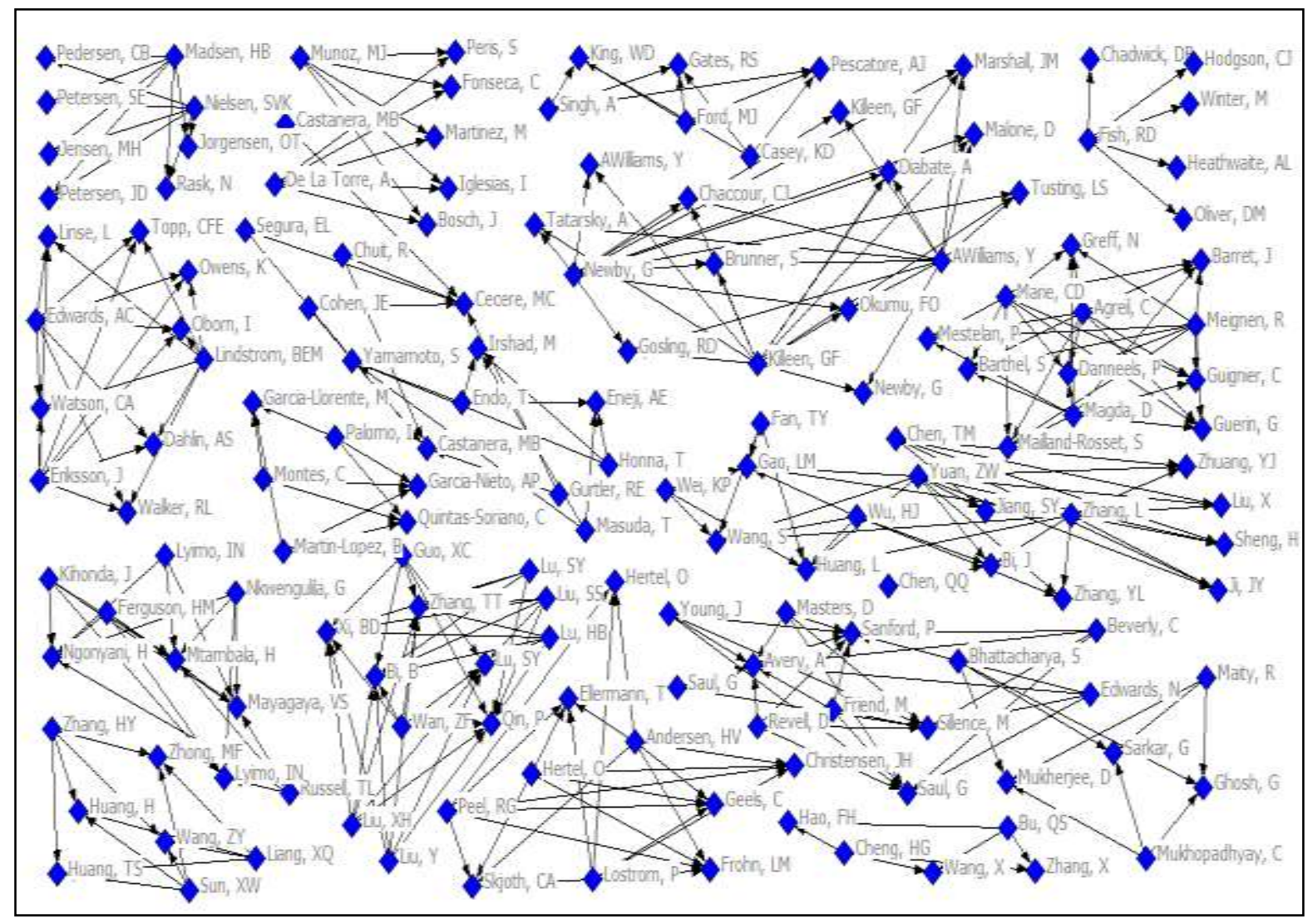

Fonte: Elaborado com o auxílio do software UCINET (2019).

Observa-se que alguns autores possuem uma rede social de cooperação superior aos demais. Como justificativa tem-se a criação de grupos de pesquisa, em sua maioria interinstitucional e em menor proporção, internacional. Destaca-se ainda a relevância deste tipo de análise em temáticas consideradas pertinentes à distintas áreas do conhecimento, sendo, portanto, dotadas de multidisciplinariedade.

Destaca-se que o principal autor sobre gestão ambiental na atividade pecuária, Z. W. Yuan, possui uma extensa rede de colaboração, integrando pesquisadores preponderantemente afiliados a sua própria instituição. Não obstante, os departamentos ou setores nos quais tais indivíduos trabalham apresentam diversificação, englobando as áreas de ciência animal, agrária e social.

\section{CONSIDERAÇÕES FINAIS}

O objetivo da pesquisa realizada foi caracterizar as publicações científicas sobre gestão ambiental na atividade pecuária a partir de métricas da informação. Para isso, utilizaram-se técnicas bibliométricas e sociométricas, pautadas nas suas leis fundamentais. 
e-ISSN: 2317-675X | Comun. \& Inf., Goiânia, GO, v. 24, p. 1-16, 2021.

Os resultados obtidos possibilitaram identificar padrões em relação às publicações acerca de uma temática que permeia distintas áreas do conhecimento e, ao mesmo tempo, circunscreve uma gama de outros assuntos. Ademais, verificou-se a maximização do interesse dos pesquisadores sobre o assunto ao longo dos últimos anos, bem como as extensas redes de colaboração, inclusive interinstitucional e internacional, a partir das quais os estudos são desenvolvidos.

Sob esta perspectiva, destaca-se a qualidade dos estudos analisados e seu consequente contributo para a ciência, o que é claramente apontado pela qualificação dos periódicos científicos por meio dos quais as investigações são divulgadas. Assim, salienta-se a relevância da pesquisa realizada, haja vista a compilação de um portfólio de artigos que abordam uma temática multidisciplinar e multifacetada. Além disso, a importância do estudo se estende também pela utilização de métricas da informação para analisar uma temática que, originalmente, provém da área de ciência animal e ciências sociais, nas quais este tipo de análise não é comumente empregado.

Contudo, reconhecem-se as limitações do estudo quanto ao não aprofundamento do conteúdo dos artigos, limitando-se à mensuração de determinados indicadores científicos. Assim, para investigações futuras sugere-se a realização de uma revisão sistemática desta literatura a fim de identificar novos mecanismos de agrupamento ou ainda novos padrões em sua elaboração, bem como confirmar as abordagens predominantes observadas por meio da estratificação das palavras-chaves.

\section{REFERÊNCIAS}

ADAMIC, L. A.; HUBERMAN, B. A. Zipf's law and the Internet. Glottometrics, v. 3, n. 1, p. 143-150, 2002.

ALEKSANDR, B.; EDUARD, V.; NATALIA, K.; EKATERINA, S.; IGOR, S.; SERGEI, L. Environmental assessment of livestock farms in the context of BAT system introduction in Russia. Journal of Environmental Management, v. 246, p. 283-288, 2019.

BARABASI, A. L.; JEONG, H.; NEDA, Z.; ASTÚCIA, E.; SCHUBERT, A.; VICSEK, T. Evolution of the social network of scientific collaborations. Physica A-Statistical Mechanics and its Applications, v. 311, n. 3-4, p. 590-614, 2002.

BLUMBERG, H. H.; HARE, A. P. Sociometry applied to organizational analysis: A review. Journal of Group Psychotherapy, Psychodrama and Sociometry, v. 52, n. 1, p. $15,1999$.

BORDONS, M.; APARICIO, J.; GONZÁLEZ-ALBO, B.; DÍAZ-FAES, A. The relationship 
e-ISSN: 2317-675X | Comun. \& Inf., Goiânia, GO, v. 24, p. 1-16, 2021.

between the research performance os scientists and their position in co-autorship networks in three fields. Journal of Informetrics, v. 9, n. 1, p. 135-144, 2015.

BRADFORD, S. C. Sources of information on specific subjects. Engineering,v. 137, p. 8586, 1934.

BRANDAU, R.; MONTEIRO, R.; BRAILE, D. M. Importância do uso correto dos descritores nos artigos científicos. Brazilian Journal of Cardiovascular Surgey, v. 20, n. 1, p. 7-14, 2005.

BRYMAN, A. Quantity and quality in social research. London: Routledge, 1988.

CHUNG, K. H.; COX, R. A. K. Patterns of Productivity in the Finance Literature: a study of the bibliometric distributions. The Journal of Finance, v. 45, n. 1, p. 301-309, 1990.

ELKINGTON, J. Sustentabilidade: canibais com garfo e faca. São Paulo: M. Books do Brasil, 2012.

FAO. The future of food and agriculture: trends and challenges. Rome: FAO, 2017.

GARFIELD, E. Citation indexes for science: a new dimension in documentation through association of ideas. International Journal of Epidemiology, v. 35, n. 5, p. 1123-1127, 2006.

GENG, S.; WANG, Y.; ZUO, J.; ZHOU, Z.; DU, H.; MAO, G. Building life cycle assessment research: A review by bibliometric analysis. Renewable and Sustainable Energy Reviews, v. 76, p. 176-184, 2017.

GIANNI, M.; GOTZAMANI, K.; TSIOTRAS, G. Multiple perspectives on integrated management systems and corporate sustainability performance. Journal of Cleaner Production, v. 168, p. 1297-1311, 2017.

PALHARES, J. C. P. Gestão ambiental nas cadeias produtivas animais. In: GEBLER, L.; PALHARES, J. C. P. (Eds.). Gestão ambiental na agropecuária. Brasília: Embrapa Informação Tecnológica, 2007.

GRIFFITHS, T. L.; STEYVERS, M. Finding scientific topics. Proceedings of the National Academy of Sciences, v. 101, p. 5228-5235, 2004.

GUTIÉRREZ, J. H.; ASTUDILLO, C. A.; BALLESTEROS-PÉREZ, P.; MORA-MELIÀ, D.; CANDIA-VÉJAR, A. The multiple team formation problem using sociometry. Computers \& Operations Research, v. 75, p. 150-162, 2016.

HOOD, W.; WILSON, C. The literature of bibliometrics, scientometrics, and informetrics. Scientometrics, v. 52, n. 2, p. 291-314, 2001.

HUAI, C.; CHAI, L. A bibliometric analysis on the performance and underlying dynamic patterns of water security research. Scientometrics, v. 108, n. 3, p. 1531-1551, 2016.

KHASSEH, A. A.; SOHEILI, F.; CHELAK, A. M. An author co-citation analysis of 37 years 
e-ISSN: 2317-675X | Comun. \& Inf., Goiânia, GO, v. 24, p. 1-16, 2021.

of iMetrics. The Electronic Library,v. 36, n. 2, p. 270-285, 2018.

LIJÓ, L.; FRISON, N.; FATONE, F.; GONZÁLEZ-GARCÍA, S.; FEIJOO, G.; MOREIRA, M. T. Environmental and sustainability evaluation of livestock waste management practices in Cyprus. Science of the Total Environment, v. 634, p. 127-140, 2018.

MARCONI, M. A.; LAKATOS, E. M. Fundamentos da metodologia científica. São Paulo: Atlas, 2011.

MILOJEVIC, S.; LEYDESDORFF, L. Information metrics (iMetrics): a research specialty with a socio-cognitive identity?.Scientometrics, v. 95, n. 1, p. 141-157, 2013.

MOURI, G.; AISAKI, N. Using land-use management policies to reduce the environmental impacts of livestock farming. Ecological Complexity, v. 22, p. 169-177, 2015.

PEHAR, F. From statistical bibliography to bibliometrics: development of a quantitative approach to the research of the written word. Libellarium: Journal for Research of Writing, Books, and Cultural Heritage Institutions, v. 3, n. 1, p. 1-28, 2010.

PINTO, G. M. C.; PEDROSO, B.; MORES, J.; PILATTI, L.; PICINI, C. T.Environmental Management Practices in industries of Brazil, Russia, India, China and South Africa (BRICS) from 2011 to 2015. Journal of Cleaner Production, v. 198, p. 1251-1261, 2018.

PODSAKOFF, P. M.; MACKENZIE, S. B.; BACHARACH, D. G.; PODSAKOFF, N. P. The influence of management journals in the 1980s and 1990s. Strategic Management Journal, v. 26, p. 473-488, 2005.

QIAN, Y.; SONG, K.; HU, T.; YING, T. Environmental status of livestock and poultry sectors in China undercurrent transformation stage. Science of the Total Environment, v. 622-623, p.702-709, 2018.

RAMANKUTTY, N.; MEHRABI, Z.; WAHA, K.; JARVIS, L.; KREMEN, C.; HERRERO, M.; RIESEBERG, L. Trends in Global Agricultural Land Use: Implications for Environmental Health and Food Security. Annual Review of Plant Biology, v. 69, n. 14, p. 14.27, 2018.

ROUSSEAU, B.; ROUSSEAU, R. Percolation as a model for informetric distributions: fragment size distribution characterized by Bradford curves. Scientometrics, v. 47, p. $195-$ 206, 2000.

SENGUPTA, I. N. Bibliometrics, informetrics, scientometrics and librametrics: an overview. Libri, v. 42, n. 2, p. 75-98, 1992.

SILVA, D. B. Sustentabilidade no Agronegócio: dimensões econômica, social e ambiental. Comunicação \& Mercado, v. 1, n. 3, p. 23, 2012.

SILVA, J. A.; BIANCHI, M. L. P. Cientometria: a métrica da ciência. Paidéia, v.11, n. 21, p. 5-10, 2001.Disponível em: http://www.periodicos.usp.br/paideia/article/view/46613/50370

SOUZA FILHO, W.; NUNES, P. A. A.; BARRO, R. S.; KUNRATH, T. R.; ALMEIDA, G. 
M.; GENRO, T. C. M.; BAYER, C.; CARVALHO, P. C. F. Mitigation of enteric methane emissions through pasture management in integrated crop-livestock systems: Trade-offs between animal performance and environmental impacts. Journal of Cleaner Production, v. 213, p. 968-975, 2019.

THOMPSON, D. F.; WALKER, C. K. A descriptive and historical review of bibliometrics with applications to medical sciences. Pharmacotherapy, v. 35, n. 6, p.551-559, 2015.

TULLO, E.; FINZI, A.; GUARINO, M. Review: Environmental impact of livestock farming and Precision Livestock Farming as a mitigation strategy. Science of the Total Environment, v. 650, p. 2751-2760, 2019.

VAN ECK, N. J.; WALTMAN, L. VOSviewer Manual. CWTS Meaningful Metrics, Universiteit Leiden, 2019.

WANG, S.; YANG, L.; SU, M.; MA, X.; SUN, Y.; YANG, M.; ZHAO, P.; SHEN, J.; ZHANG, F.; GOULDING, K.; SHI, X.; LIU, X. Increasing the agricultural, environmental and economic benefits of farming based on suitable crop rotations and optimum fertilizer applications. Field CropsResearch, v. 240, p. 78-85, 2019.

ZANONI, M. A questão ambiental e o rural contemporâneo. Desenvolvimento e Meio Ambiente, v. 10, p. 101-110, 2004. 